\title{
Los seguros marítimos y la movilidad como biopolítica de seguridad
}

\section{Maritime insurance and mobility as biopolitics of security}

\author{
Luis LOBO-GUERRERO \\ Royal Holloway, University of London \\ Luis.Lobo-Guerrero@rhul.ac.uk
}

Recibido: 16.02 .2012

Aprobado definitivamente: 02.10 .2012

\section{RESUMEN}

Regímenes de movilidad no son en absoluto naturales. Son el resultado de complejos entramados de poder que incorporan formas de control de comportamientos, a manera de lo que se puede llamar economías morales. El analizar la movilidad como fenómeno político, social, cultural, y económico, circunscrito a la promoción y protección de formas y estilos de vida, abre la posibilidad de investigar lo que puede llamarse, siguiendo el pensamiento de Foucault, una biopolítica de movilidad. La movilidad, desde está perspectiva, debe investigarse no desde lo abstracto sino en relación a prácticas, discursos, y tecnologías concretas a través de las cuales personas, bienes y servicios son puestas en circulación. Tal circulación, junto con la conectividad que la acompaña y la complejidad de redes que se tejen en su articulación, constituyen lo que ha denominado como los cuasi-transcendentes de la vida liberal contemporánea. Elaborando esta teoría, este artículo ofrece una lectura del fenómeno de la movilidad oceánica a partir del estudio de discursos y prácticas de seguros marítimos. En concreto, se analiza el rol de Lloyd's of London como mercado global de seguros marítimos en la articulación de economías morales de movilidad oceánica.

Palabras Clave: Biopolítica, riesgo, circulación, seguros marítimos, seguridad internacional, movilidad

\begin{abstract}
There is nothing natural about mobility regimes. They are the result of complex ensembles of power that involve the control of conducts in the form of moral economies. To analize mobility as a political, social, cultural and economic phenomenon circumscribed to the promotion and protection of forms and styles of life, opens up the possibility to investigate, following the work of Foucault, what can be called a biopolitics of mobility. Mobility, from this perspective, must be approached, not from the abstract, but from concrete practices, discourses and technologies through which people, goods, and services are put in circulation. Such circulation, together with its allied connectivity and complexity, constitute what has been termed the quasi-transcendentals for contemporary liberal life. Building on this theory, the article offers a reading of maritime mobility from the study of insurance discourses and practices. In particular, the role of Lloyd's of London is analyzed as constituting moral economies of maritime mobility.
\end{abstract}

KEYwORDS: Biopolitics, risk, circulation, maritime insurance, international security, mobilities

\section{SUMARIO}

1. Hacia una biopolítica de seguridad de los seguros marítimos. 2. La piratería como obstrucción de la movilidad y la producción de imaginarios de seguridad oceánica. 3. Asegurando la circulación oceánica como espacio heterotópico. 4. Conclusiones. 
Desde la década de 1970 y a partir del trabajo de Michel Foucault (p.e. Foucault, 1989, Foucault, 1991, Foucault, 1998 , Foucault, 2003, Foucault, 2004b, Foucault, 2004a, Foucault, 2008, Foucault, 1964, Foucault, 2001) numerosos estudios se han adelantado acerca de lo que se puede entender como los fenómenos biopolíticos de la era moderna. Estos se pueden caracterizar de manera general a partir de las formas de saber, de los discursos y prácticas que de ellas derivan y de su materialización en formas de promover y proteger 'la vida'. En el plano de la teoría política se han generado, por ejemplo, estudios sobre las maneras en las cuales individuos y poblaciones son constituidas como objetos y sujetos de gobierno y las formas de poder ejercidas para hacer esto posible (p.e. Agamben, 1998, Barry, 2001, Dillon and Lobo-Guerrero, 2009, Foucault, 1998 , Foucault, 2003, Foucault, 2004b, Foucault, 2008, Hardt and Negri, 2001, Hardt and Negri, 2004, Rose, 1999, Esposito, 2012, Mendiola Gonzalo, 2009)1. Se ha enfatizado de manera particular el análisis de las condiciones de posibilidad y las condiciones de operabilidad que soportan las tecnologías de poder desarrolladas para la promoción de particulares estilos de vida (el 'hacer vivir'). También se ha prestado atención a las prácticas dirigidas a la protección de esas formas de vivir, prácticas que incluyen en muchos casos el 'dejar morir' o de vivir 'de forma paralela' como por ejemplo la concentración de individuos en asilos, la operación de campos de internamiento, campamentos de refugiados y de zonas de control migratorio. Sin embargo, poco énfasis se ha dado al estudio de las prácticas biopolíticas que producen espacios de circulación, particularmente de circulación oceánica.
De forma muy general es posible clasificar estos estudios alrededor de lo que Foucault presentó como los cuasi-trascendentes de la vida humana; en el caso que ocupa este artículo, de la vida liberal. Estos referentes fueron identificados a partir del estudio de las ciencias de la vida, de las ciencias de la economía y de los saberes del lenguaje (la vida, el trabajo y la lengua) (Foucault, 1989: 272-329). De estas tres esferas las ciencias de la vida se han convertido en el espacio empírico primordial en el estudio de la biopolítica aunque significativos avances teóricos se han hecho en áreas de la economía política y de las ciencias del lenguaje.

El imaginario biopolítico (ver Dillon and LoboGuerrero, 2009), sin embargo, se refleja en fenómenos mundanos y cercanos a la vida diaria como lo es la movilidad. Este fenómeno constituye un espacio empírico que abre la posibilidad de entender la vida como expresión de formas de poder que a su vez proceden a partir de ciertas tecnologías. La movilidad está referida al imaginario biopolítico en formas tan tangibles y reales como por ejemplo el comercio internacional. Los estilos de vida y el sustento de los individuos y colectividades que dependen de este tipo de comercio, primordialmente en países de economías liberales avanzadas, son posibles a partir de tecnologías como los seguros, un tema que ha recibido poco interés por parte de investigadores en temas biopolíticos. ${ }^{2}$ La movilidad como efecto biopolítico está influenciada de manera directa por formas de seguros (e.g. marítimo, incendio, vida) que promueven la circulación de individuos, colectividades, bienes, ideas y servicios. La complejidad y la conectividad que resulta de la circulación de estos elementos se ve reflejada en el diseño, la operación

\footnotetext{
${ }^{1}$ La bibliografia en estos temas es bastante voluminosa y lo mencionado es apenas un ejemplo. Un compendio sobre material recientemente producido en esta area puede encontrarse en la pagina web de la Red Biopolitica: http://www. biopolitica.cl, en la seccion: Biblioteca.

${ }^{2}$ Los siguientes trabajos son ejemplos de investigaciones en este tema: EWALD, F. 1991. Insurance and Risk. In: BURCHELL, G., GORDON, C. \& MILLER, P. (eds.) The Foucault Effect: Studies in Governmentality, with two lectures and an interview with Michel Foucault. Chicago: The University of Chicago Press 197-210, ERICSON, R., DOYLE, A. \& BARRY, D. 2004. Insurance as Governance, Toronto, University of Toronto Press, CLARK, G., ANDERSON, G., THOMANN, C. \& GRAF VON DER SCHULENBURG, J. M. 2010. The Appeal of Insurance, Toronto, University of Toronto Press, BAKER, T. 2000. Insuring Morality. Economy and Society, 29, 559-577, BAKER, T. \& SIMON, J. 2002. Embracing Risk. In: BAKER, T. \& SIMON, J. (eds.) Embracing Risk: The Changing Culture of Insurance and Responsibility. Chicago: University of Chicago Press 1-26, LOBO-GUERRERO, L. 2011. Insuring Securtity: Biopolitics, Security and Risk, Abingdon, Ox., Routledge, LOBO-GUERRERO, L. 2012a. Insuring War: sovereignty, security and risk, Abingdon, Ox., Routledge.
} 
y las prácticas de aseguramiento al igual que en los mercados que derivan de ellos. Si bien es cierto que la vida liberal se caracteriza incrementalmente por la tecnologización y la comodificación de las ciencias de la vida, de la constitución de economías políticas basadas en la promoción y protección del vivir, y de la comunicación activa de la existencia, es necesario ensanchar el análisis del imaginario biopolítico liberal a la mundanidad del comercio internacional y de las tecnologías que hacen posible la circulación oceánica si se ha de entender la biopolítica como efecto empírico de la existencia.

En este artículo se propone que las relaciones de circulación y conectividad que caracterizan la movilidad oceánica sobre la cual depende el comercio internacional, y una forma global de vida liberal, es facilitada de forma directa por la acción de los seguros marítimos. Estos seguros tienen como efecto una forma de poder que produce imaginarios de seguridad, entendidos estos como espacios de navegación comercial ininterrumpida. A manera de hacer visible y explícito el rol de estos seguros y de los mercados que los suscriben, la movilidad oceánica es analizada en este artículo a partir de una de sus obstrucciones, la piratería marítima. Se arguye, por medio de una descripción detallada de la forma en la cual un mercado de seguros como lo es Lloyd's of London ejerce un rol de mayorazgo en los mares del mundo, que los seguros marítimos ejercen una biopolítica de movilidad con claros efectos en el gobierno de los barcos, de sus tripulaciones, sus dueños y operadores. Al gobernar los buques, aseguradores en Lloyd's ejercen un gobierno sobre espacios heterópicos constituyendo un orden de navegación marítima global. Esta forma de gobierno tiene implicaciones para el estudio de la biopolítica y la movilidad que se hacen explícitas en la conclusión final.

\section{HACIA UNA BIOPOLÍTICA DE MOVILIDAD DE LOS SEGUROS MARÍTIMOS}

La piratería marítima contemporánea es utilizada en este artículo como espacio empírico a partir del cual se empieza a hacer evidente la operación de los seguros marítimos como tecnología de poder enfocada a promover y proteger la circulación y conectividad oceánica sobre la cual depende el comercio internacional. Por su silenciosa acción esta tecnología usualmente pasa desapercibida para los investigadores en temas sociales, económicos y políticos aunque constituye un espacio privilegiado desde el cual estudiar relaciones de poder. El enfoque del artículo es el orden que resulta del ejercicio de ese poder, un orden que por lo imperceptible, es tradicionalmente incuestionado. Aunque los seguros se han presentado históricamente como indispensables para la navegación marítima (e.g. Weskett, 1781, Park, 1817, Harvard Law Review, 1920, Rose, 2004), poca crítica e historia se ha escrito sobre la racionalidad en la que operan.

La tecnología de riesgo que subyace la operación de los seguros marítimos opera bajo la promesa de proveer compensación por la pérdida de capital que pueda resultar de la interrupción en la circulación de buques y carga o de la pérdida total o parcial de los mismos. Al hacerlo, los seguros facilitan la conectividad entre centros productivos, centros de materias primas y nodos portuarios alrededor del globo. Cuando buques mercantes que han sido asegurados son sacados de circulación por las acciones 'piráticas' de un grupo de individuos, la acción de los seguros como facilitador de la movilidad global empieza a revelarse.

En principio, no hay nada nuevo en revelar la existencia de los seguros marítimos ya que su historia se remonta a tiempos del Rey Hammurabi de Babilonia (c. 1750 A.C.) (ver Trenerry, 1926). Lo que si es de reciente desarrollo son las estrategias de poder que se han articulado durante la primera década del siglo XXI desde los mercados de seguros marítimos para enfrentar la piratería marítima. Estas estrategias, como se detallará más adelante, permiten presentar a los seguros marítimos como una tecnología biopolítica que influye de manera estratégica en el comportamiento de individuos y colectividades dedicadas al comercio internacional. Como tal, los seguros marítimos son un actor fundamental en la constitución de economías morales en el negocio de la navegación marítima.

Lloyd's of London es el más antiguo de estos mercados y el más importante por la concentración de experticia técnica y regional que le permite mantener operaciones a nivel global. Contrario a lo que usualmente se asume, Lloyd's no es una compañía de seguros sino un mercado ubicado en el centro financiero de Londres. Como mercado, concentra 
en su seno una gran variedad de capital, saberes y experiencia lo que le permite no solo innovar en los productos sino también adaptarlos a las circunstancias cambiantes. Su membresía es limitada y los miembros, que pueden ser personas y consorcios, no se limitan a la suscripción de seguros marítimos. También suscriben seguros sobre riesgos especiales (como aquellos relacionados con satélites), al igual que otras formas de seguro y reaseguro. La operación del mercado se organiza alrededor de una franquicia que se encarga de administrar los servicios comunes y generar las reglas internas del mercado. La franquicia opera también comités especializados a partir de los cuales se producen lineamientos que en algunos casos obligan y en otros informan a los miembros sobre condiciones especificas del mercado. Como se explicará más adelante, uno de esos comités es el de Guerra que se encarga de monitorear cambios en el ambiente político internacional que puedan afectar las condiciones de riesgo que se aseguran en el mercado. Debido a la importancia que las decisiones tomadas en Lloyd's tienen para el comercio global y la estabilidad internacional, Lloyd's se ha empezado a analizar como un actor internacional no estatal y no gubernamental (Haufler, 1993, Haufler, 1997, Lobo-Guerrero, 2012a). Su estructura y operaciones le permiten ejercer presión a nivel global sobre estados y actores privados con el fin de mantener y establecer orden en los mares y océanos del mundo. Por medio de esa presión, Lloyd's genera geografías de seguridad que son difíciles de contemplar a partir de las concepciones terrestres que han caracterizado disciplinas como las Relaciones Internacionales y la Geografía Humana.

Como se explicará luego, la presión de Lloyd's se ha constituido en una forma de poder con gran injerencia internacional. Sus efectos tienen claras connotaciones geopolíticas cuando estados soberanos moldean su conducta en función de los dictámenes de este mercado. Este fue el caso, por ejemplo, cuando Lloyd's intervino para reducir la piratería en el Estrecho de Malaca a mediados de la década del 2000 (Lobo-Guerrero, 2008), un caso con repercusiones internacionales y que ha sentado un precedente importante para el análisis de la gobernanza global. Al mismo tiempo, los efectos del poder de Lloyd's son también biopoliticos ya que afectan directamente a una forma particular de vida, un estilo de vida liberal cuyo sustento depende de la capaci- dad de comerciar ininterrumpidamente a través de los mares. El caso de la piratería en el Océano Indico y el Golfo de Adén, como se ilustrara mas adelante, es ejemplo de ello (Lobo-Guerrero, 2012b).

La forma de poder ejercida desde Lloyd's no encuadra dentro de las tipologías generales del poder desarrolladas tradicionalmente desde la Ciencia Política. En un esfuerzo por teorizarla se ha presentado como un 'mayorazgo' (stewardship) de la circulación marítima global (Lobo-Guerrero, 2011: 108122). Mayorazgo no se entiende en este contexto como la figura del antiguo derecho castellano (e.g. Escriche, 1840). Siguiendo una de las interpretaciones anglosajonas de este término (ver Steinberg, 2001) se percibe como el ejercicio de una forma de poder que regula la capacidad de acceder y permanecer, en este caso, dentro de la circulación oceánica.

Es importante anotar también que la circulación marítima global es analizada en el contexto de este trabajo, no bajo la categoría de objeto, sino como cuasi-transcendente espacial a partir del cual se puede explorar el fenómeno de la seguridad oceánica. Esta posición está centrada en el trabajo de Foucault quien observó cómo la categoría de cuasi-transcendente siempre se da en la forma de una revelación (Foucault, 1989: 397). No es objeto en sí mismo pero revela la operación de entramados de poder a partir de los cuales se descubre el fenómeno. El rol de mayorazgo en este caso implica la regulación de flujos a partir de la autorización para circular (navegar), no de forma soberana como sucede cuando esto ocurre a partir de las estructuras político-jurídicas del derecho publico europeo (Schmitt, 2003, Schmitt, 2011). El mayorazgo es ejercido por medio del gobierno de un imaginario político de 'lo incierto' el cual es bastante particular. A partir de este imaginario operan tecnologías de riesgo como lo son los seguros (Ewald, 1991, Lobo-Guerrero, 2011, Clark et al., 2010). Esta forma de gobierno constituye lo que, siguiendo a Foucault, se entiende como el gobierno de conductas a distancia (e.g. Dean, 1999a, Burchell, 1996).

Uno de los principales efectos del mayorazgo que Lloyd's ejerce sobre la circulación marítima global es la proyección de su imaginario de seguridad a través de las pólizas de seguros que se suscriben en su mercado. El ejercicio de está forma de poder tiene implicaciones geográficas (e.g. en el diseño y operación de las rutas mercantes), materiales 
(e.g. en el diseño y operación de los buques y de las medidas de operación necesarias para protegerlos), políticas (e.g. en el comportamiento de estados soberanos con relacion a la protección del comercio marítimo), económicas (e.g. en el precio de las pólizas de seguros, de los bienes transportados, y de la viabilidad de las economías locales), y morales (e.g. en el comportamiento de propietarios, operadores y tripulaciones y en la regulación de los mercados de seguros). Tales implicaciones se materializan en la constitución de un 'espacio' de gobierno que no corresponde a los espacios tradicionalmente analizados por las Relaciones Internacionales, el Derecho Internacional, y la Geografía Humana. Siguiendo la tradición analítica de Foucault, quien argumentó que el barco (la nave) es la heterotopía por excelencia (1997), se demostrará en la tercera sección de este artículo cómo el ejercicio del poder que subyace los discursos y prácticas aseguradoras del mercado de Lloyd's, implica en su conjunto la producción de 'otros' espacios de circulación. En tanto heterópicos, estos espacios constituyen nuevas dimensiones desde las cuales se puede estudiar la producción de la seguridad marítima. En la articulación de estos espacios de circulación intervienen complejos de poder/saber por medio de los cuales, en su afán por restablecer la circulación a través de los cuellos de botella geográficos que utilizan los piratas para facilitar su accionar, los aseguradores marítimos diseñan estrategias para sopesar el riesgo que sus clientes les representan. Al analizar esas estrategias es posible describir el entramado de poder asegurador sobre el cual depende más del ochenta por ciento del comercio global (MIU, 2007).

\section{LA PIRATERÍA COMO OBSTRUCCIÓN DE LA MOVILIDAD Y LA PRODUCCIÓN DE IMAGINARIOS DE SEGURIDAD OCEÁNICA}

De acuerdo con un comunicado de prensa emitido por Lloyd's of London, la piratería marítima en el Océano Índico amenaza con 'estrangular' vías marítimas que son cruciales para la economía global y cuyos costes ascienden por el momento a doce millones de dólares al año (Lloyd's of London, 2011).

A partir del ano 2004 se ha observado un crecimiento acelerado del fenómeno de la piratería marítima en varias partes del globo, particularmente en el Golfo de Adén y el Océano Índico (IMB, 2011). A principios de la década del 2000 este fenómeno fue visto como un problema doméstico localizado principalmente en el área del Estrecho de Malaca en el sureste asiático (Young, 2007). Con el avance de la década, el problema se consolidó en las costas de Somalia (Marchal, 2011) y se convirtió en un obstáculo para la movilidad oceánica global con consecuencias económicas de gran importancia (Murphy, 2010).

La piratería marítima está lejos de ser un fenómeno en retroceso. Por el contrario, de acuerdo con cifras del Bureau Marítimo International (IMB), 1181 personas fueron capturadas en hechos de piratería durante el 2010. Ese mismo año, cincuenta y tres buques fueron capturados lo cual constituye un récord en lo que viene caracterizándose como una nueva economía en la sombra [shadow economy] (e.g. Odeke, 2011). El modelo de negocio sobre el cual el fenómeno se desarrolla no es estático. Aunque es difícil de definir como un solo fenómeno, ya que involucra un conjunto de conflictos y prácticas económicas, se caracteriza por una gran capacidad de adaptación a las cambiantes circunstancias. Por ejemplo, a mediados de la primera década del siglo, cuando el epicentro del problema se ubicaba en el Estrecho de Malaca, el modelo pirata se concentraba en abordar buques pequeños para robar dinero y algunos bienes de a bordo y desaparecer rápidamente. Este modelo suponía una base de soporte logístico y político en las costas del litoral donde se encuentra el Estrecho de Malaca, o en las costas de Nigeria. Hacia el final de la década, sobre todo con el desarrollo de la piratería en las costas de Somalia, los llamados 'piratas' avanzaron hacia un nuevo modelo que involucra la captura de buques de cada vez mayor tamaño para cobrar un rescate en dinero. Este nuevo modelo implica un mayor y más complejo apoyo político, social, y logístico que incluye el uso de buques nodriza, comunicaciones más sofisticadas, y apoyo económico para financiar la empresa y las maniobras financieras a nivel internacional que se requieren para recibir y procesar los rescates. Sobre todo, como lo relata Marchal (2011), este esquema cuenta con el apoyo de comunidades somalíes que han visto como la comunidad internacional ha afectado la pesca local sobre la cual se sostenía la economía de la región. 
Esta forma de piratería empieza a evidenciar también una transformación en su modelo de negocio. En los últimos años se ha observado que de manera creciente las tripulaciones se han convertido en objeto mismo de secuestro. Los capitanes de los barcos y miembros de las tripulaciones son retenidos con el objeto de ejercer presión en la negociación sobre la liberación del buque y su carga. La vida misma de estas personas se ha convertido en objeto de negocio dándose casos en los que se negocian por separado las vidas y el buque con su carga. Esto, en palabras de Neil Smith, director de suscripción de seguros del mercado de Lloyd's (LMA), 'ha cambiado las reglas del juego' (Lloyd's of London, 2011).

La cifras del negocio también demuestran cambio. En el año 2005 los rescates demandados eran estimados en 150,000 dólares americanos. En el 2011 el promedio se estimó en 5.4 millones, de acuerdo con la organización Oceans Beyond Piracy (Lloyd's of London, 2011). Estos incrementos están relacionados con el desarrollo de nuevos teatros de operaciones piratas. Por ejemplo, el 9 de febrero de 2011 fue secuestrado el buque Irene SL cargado con dos millones de barriles de petróleo originados en Kuwait y destinados al mercado de los Está dos Unidos. La carga fue estimada en doscientos millones de dólares equivalente a un quinto de la producción diaria de ese país. La captura del buque, el más grande y costoso hasta ese momento, tuvo lugar a novecientas millas de la costa Somalí. El hecho impresionó a la industria mercante y aseguradora ya que demostró que el problema dejó de ser asunto de mares territoriales y se convirtió en asunto de mar abierto (Wright, 2011).

La respuesta política internacional a este problema hasta el 2010 había asumido la forma de una estrategia naval internacional de acuerdo a lineamientos dictados por la Organización de Naciones Unidas. La Resolución 1851 del Consejo de Seguridad autorizó a fuerzas navales internacionales el adelantar operaciones anti-piratería en las aguas territoriales de Somalia al igual que operaciones terrestres en las costas de ese país, siempre y cuando se contara con el consentimiento del Gobierno Fed- eral de Transición Somali (GFTS). La Resolución 1872 autorizó la participación de países miembros de las Naciones Unidas en el entrenamiento y equipamiento de las fuerzas de seguridad del GFTS durante un período de doce meses comenzando en Mayo de 2009, término que fue extendido por un año más. Dentro del marco propiciado por la Resolución 1851, la Unión Europea inició en diciembre de 2008 la primera operación de su Política Común de Defensa y Seguridad cuya misión es la de cubrir un área marítima comparable con el tamaño del Mar Mediterráneo (House of Lords EU Committee, 2010: 7).

La industria mercante internacional trabaja sobre la idea de que las fuerzas navales internacionales deben concentrar sus operaciones en la detección y ataque a las naves nodrizas desde las cuales se lanzan los ataques piratas y en la reacción militar para rescatar naves y tripulaciones que hayan sido capturadas. El personal armado a bordo de los buques debería en principio contribuir a la disuasión de los ataques ya que hasta Octubre de 2011 ningún buque con escolta a bordo había sido capturado (BBC News, 2011). Sin embargo, la industria aseguradora aún no logra un consenso sobre si aceptar o no la utilización de personal armado a bordo de las naves.

Medidas como estas contribuyen a reforzar la presentación del problema como uno de seguridad entendido a partir de la defensa armada. El debate público se ha concentrado principalmente en determinar cómo, por medio de la fuerza, se puede controlar la piratería. La presión ejercida por el sector asegurador, sin embargo, evidencia otra historia y otra forma de poder, en este caso, biopolítico. Por un lado, la solución militar al problema es limitada. El Capitán (lieutenant-commander) Jimmie Adamsson de la fuerza naval europea Navfor, encargada de patrullar las aproximaciones al Estrecho de Adén, lo ha expresado de la siguiente manera. 'Es como apretar un globo de goma', 'si se aprieta sobre el Golfo de Aden, el globo se expandirá hacia otras direcciones donde no estemos nosotros' (Wright, 2011). Por el otro, se viene comentando que el admitir personal armado a bordo de los buques, recurriendo a la al-

\footnotetext{
${ }^{3}$ En relación a las limitaciones del uso de compañias militares privadas, ver LEANDER, A. 2010. The Paradoxical Impunity of Private Military Companies: Authority and the Limits to Legal Accountability. Security Dialogue, 41, 467-490.
} 
ternativa de emplear personal de la industria militar tanto pública como privada ${ }^{3}$, repercutirá en el escalamiento del conflicto forzando la utilización de armas de mayor calibre y el desarrollo de tácticas y estrategias logísticas y militares más complejas.

Dadas las características del problema, una lógica militar que depende de la identificación, administración y destrucción de la amenaza, es limitada en su alcance. Implica la definición del enemigo y el tratamiento del mismo de acuerdo con la doctrina militar, las reglas de enfrentamiento, los lineamientos políticos y las reglas internacionales de la guerra. En el caso de la piratería marítima se trata de actores no internacionales constituidos por grupos de individuos que por ciertas circunstancias sociales, económicas y políticas operan un modelo de negocio que busca capturar bienes en el mar con el objeto de obtener rescate. Las fuerzas navales no son adecuadas para enfrentar las estrategias y tácticas empleadas por estos grupos. La industria aseguradora las ve tan solo como parte de una estrategia que permite asumir el problema en términos de riesgo.

La lógica de la industria se preocupa de la identificación y la administración del riesgo generado por las prácticas piráticas en relación a la circulación marítima global la cual es entendida como elemento fundamental para el comercio internacional. Dejando de lado las connotaciones sociales y económicas de los llamados piratas, el asunto adquiere para los aseguradores una perspectiva de obstrucción a la circulación marítima. Vista de está forma, la piratería deja de ser un asunto de estados, regulado a través del derecho internacional y del derecho del mar, y se convierte en un problema logístico-comercial. Desde esta perspectiva, lo que se requieren son estrategias para restablecer la operación normal de flujos comerciales para lo cual entender el problema en términos de riesgo trae ciertas ventajas. De forma rápida, ya que el tema se desvía del enfoque de este artículo, el presentar una realidad en términos de riesgo implica interpretar un fenómeno en relación a las incertidumbres que presenta. Incertidumbres son por supuesto entendidas en relación a circunstancias que se dan por ciertas (e.g. algún evento o status quo) y las tecnologías de riesgo, como los seguros, son utilizadas para transformar esas incertidumbres en algo gobernable. En trabajos previos se ha teorizado este entendimiento del riesgo como la transformación de incertidumbres en material fungible, entendiendo esto como algo que se puede comerciar e intercambiar económicamente (Lobo-Guerrero, 2011: 13-33). A través de prácticas, las compañías aseguradoras definen los eventos contra los cuales se busca seguro y suscriben pólizas cuyo coste debe representar el nivel de riesgo incurrido en la posibilidad de que el evento asegurado se materialice (p.e. la pérdida total o parcial de un buque como consecuencia de una tormenta dentro de un periodo y una región establecida). Al hacerlo, las compañías aseguradoras transforman las incertidumbres alrededor de la materialización del evento asegurado en riesgo; esto es, incertidumbre fungible.

El presentar un fenómeno en términos de riesgo, o para utilizar los términos Foucaultianos, el problematizar una situación en términos de una racionalidad de riesgo (e.g. Ewald, 1991, O’Malley, 2004, Dean, 1999b, Ericson et al., 2004), permite referirse al mismo fenómeno desde distintos ángulos con el objeto de obtener distintas soluciones. Por ejemplo, los aseguradores pueden ofrecer pólizas marítimas para proteger un buque, su tripulación y su carga y dichas pólizas pueden cubrir ataques piratas. También pueden asegurar la piratería como evento independiente como viene sucediendo desde el año 2005 en el mercado de Lloyd's cuando la asociación de suscriptores que operan allí decidió excluir la piratería de las pólizas generales conocidas como de casco y maquinaria. Desde entonces la piratería se viene suscribiendo dentro de pólizas especiales conocidas como pólizas de guerra, y al hacerlo, las condiciones de aseguramiento han cambiado (ver Lobo-Guerrero, 2012b). Por ejemplo, en lugar de asegurar un buque por períodos de un año, las pólizas de guerra se suscriben por viaje, lo cual permite a los aseguradores analizar los factores de riesgo específicos para cada trayecto. En el último ano se han empezado a suscribir pólizas anti-secuestro para cubrir a los patrones y tripulantes de los buques de manera independiente de la póliza general (una práctica, que aunque antigua, no era común hasta el ultimo quinquenio). De esta forma la industria se acomoda a las cambiantes circunstancias del fenómeno pirático y problematiza el fenómeno a través de eventos sobre los cuales se puede operar una tecnología de riesgo. De forma más general, un mercado de seguros como lo es Lloyd's también puede influenciar el ambiente político dentro del cual opera un fenómeno, como se ilustrará en la siguiente sección. Lo importante por 
el momento es resaltar que al emplear una racionalidad de riesgo con las lógicas que la hacen posible, por ejemplo la lógica actuarial y la lógica de administración de riesgo (Power, 2003, Power, 2004, Power, 2007), los aseguradores proyectan su imaginario de incertidumbre a través de una tecnología de seguridad como lo es el seguro. No es un imaginario necesario e inevitable, tan sólo uno que resulta del espíritu emprendedor que lleva al asegurador a transformar incertidumbres en riesgo como oportunidad de negocio. Cómo esos imaginarios aseguradores son alimentados, es tema para otro artículo.

\section{ASEGURANDO LA CIRCULACIÓN OCEÁNICA COMO ESPACIO HETERÓPICO}

Michel Foucault, en Los Espacios Otros, se refirió a la nave como 'la heterotopía por excelencia' como

un espacio flotante del espacio, un espacio sin espacio, con vida propia, cerrado sobre sí mismo y al tiempo abandonado a la mar infinita y que, de puerto en puerto, de derrota en derrota, de prostíbulo en prostíbulo, se dirige hacia las colonias buscando las riquezas que éstas atesoran, puede comprenderse la razón por la que la nave ha sido para nuestra civilización, desde el siglo xvi hasta hoy, al tiempo, no sólo, por supuesto, el mayor medio de desarrollo económico $[\ldots]$, sino el mayor reservorio de imaginación (Foucault, 1997).

El ejercicio de mayorazgo sobre la circulación marítima global que ejerce Lloyd's en el siglo XXI permite desplazar el planteamiento de Foucault, de la nave al cuasi-transcendente de la circulación. En este caso la heterotopía no es constituida por la nave como espacio flotante desconectado del espacio terrestre. La heterotopía es el resultado del ejercicio del poder de Lloyd's sobre la circulación marítima, un poder que se distingue de la territorialidad que caracteriza al poder de los Estados soberanos y que constituye un espacio de influencia propio. La circulación marítima, como fenómeno de la movilidad, es el resultado del ejercicio de poder sobre objetos tradicionales como el territorio y las poblaciones, pero también sobre espacios otros como la circulación marítima. Constituye, parafraseando a Foucault, 'un sistema de apertura y cierre' que de- termina qué ha de circular y cómo ha de ser tal circulación. Las decisiones de Lloyd's determinan, de forma fluida y cambiante, las condiciones de acceso y permanencia a la circulación protegida; esto es, asegurada; necesaria para la protección del capital flotante y en tránsito sobre el cual depende el comercio marítimo global. Estas acciones y el entramado de poder que resultan de ellas son en consecuencia tan importantes como lo son los acuerdos y tratados internacionales que tradicionalmente han regulado el comercio marítimo global. Por su importancia es necesario describirlas en detalle a través de casos concretos lo cual se ofrece a continuación.

El 20 de Junio de 2005, el Comité de Guerra de Lloyd's of London (Joint War Committee -JWC) tomó la decisión de incluir en su Lista de Guerra -oficialmente llamada 'Hull War, Strikes, Terrorism and Related Perils List'- al litoral donde se encuentra ubicado el Estrecho de Malaca. El estrecho opera como cuello de botella para el flujo de buques mercantes conectando las economías del Asia con el Mediterráneo y de allí con el norte de Europa, el Atlántico, y las Américas. Por su posición geográfica y debido a corrientes marinas y otros factores, Malaca es uno de los puntos geoestratégicos de mayor importancia en la navegación global.

Lloyd's of London, cuyos inicios se remontan al café que Edward Lloyd está bleció en 1691 (Gibb, 1957), y con una historia que acompaña el desarrollo del Imperio Británico hasta su disolución al final de la Segunda Guerra Mundial, ha jugado un papel esencial en la articulación de una economía global en la que Londres aun ejerce un poder financiero preponderante. Desde su consolidación como mercado de seguros hacia finales del siglo XVIII, Lloyd's ha sido un importante aliado del Estado británico en sus esfuerzos bélicos, particularmente durante las Guerras Napoleónicas y las dos Guerras Mundiales (Lobo-Guerrero, 2012a). Quizás por está razón no sorprende saber que a partir del final de la Segunda Guerra Mundial Lloyd's ha operado un Comité de Guerra, que desde finales de los anos noventa se conoce como el JWC. Es un comité compuesto por un grupo de aseguradores que forma parte de la Asociación del Mercado de Lloyd's y de la Asociación Internacional de Suscriptores y tiene como objeto analizar los riesgos de 'guerra' que puedan afectar los niveles normales de riesgo sobre los cuales se aseguran los cascos de los buques en el mercado de 
Lloyd's. El Comité de Guerra se ocupa en exclusividad de los cascos de los buques ya que se asume que al controlar la seguridad del casco se controla por ende la seguridad de la carga.

El Comité produce regularmente su Lista de Guerra en la cual se incluyen aquellos países y áreas del mundo que pueden representar un nivel elevado de riesgo para la circulación de buques. Casos como la Guerra del Golfo en 1991 ocasionaron la inclusión de Iraq y Kuwait en la Lista. Al final de la Guerra Fría, los criterios sobre los cuales decidir si un está do se encontraba en guerra o no cambiaron notoriamente y el Comité se empezó a ocupar de casos de guerra civil, terrorismo e inestabilidad política. Las consecuencias derivadas de la inclusión de un país o zona dentro de la Lista son principalmente financieras. Ni el Comité ni Lloyd's pueden forzar a sus miembros a elevar los precios de sus pólizas por cuestiones de las leyes de competencia (competition law), pero pueden recomendar a sus miembros que el circular por ciertas zonas incurre un riesgo elevado. En efecto, al incluir a un país o zona en la Lista los precios en las pólizas sobre los cascos de los barcos que naveguen hacia, desde, y a través de esas zonas tienden a incrementarse. En esos casos la diferencia es asumida por el comerciante o el transportador, o trasladada al consumidor en el precio final del producto transportado. Este factor implica que cualquier incremento en el precio de las pólizas tiene un efecto potencial en el coste de vida de las poblaciones que dependen del comercio marítimo. Tan solo pensar en los efectos que esto puede tener en los precios del petróleo permite imaginar las consecuencias a nivel global.

Las decisiones de incluir o no una región o país en la lista tienen efectos políticos. Cuando el litoral donde se encuentra el Estrecho de Malaca fue incluido en La Lista en Junio de 2005, el primer ministro de Singapur, junto con los mandatarios de Indonesia y Malasia, presentaron una queja ante el presidente del comité directivo de Lloyd's. Argumentaron que sus estados no se encontraban en guerra y que por lo tanto no entendían la decisión de incluirlos en la Lista. Lloyd's respondió con el argumento de que la inclusión no era una intromisión de tipo político, solo de tipo técnico ya que estaba soportada en un informe preparado por el Bureau Marítimo Internacional que evidenciaba un incremento de la piratería en la región con 138 ataques en el Estrecho en el ano
2004. Para contrarrestar ese argumento, el gobierno de Singapur comisionó un estudio del Instituto de Estudios Estratégicos en Londres que concluyó que la acción piratica en la región estaba en retroceso. El estudio fue utilizado por Singapur para cuestionar la decisión de Lloyd's. Sin embargo, Lloyd's sostuvo su posición e indicó que la decisión fue tomada con base a la asesoría de expertos y que hasta tanto no hubiese una reducción significativa en los ataques piratas cometidos en la región, Lloyd's recomendaría a sus miembros reflejar el incremento del riesgo de ataques piratas en la suscripción de sus pólizas. Lloyd's añadió que sus decisiones no eran de índole política sino meramente técnicas. Dos meses más tarde, en agosto de 2005 Indonesia, Malasia y Singapur firmaron un acuerdo de cooperación naval y militar para incluir elementos aéreos en el patrullaje del Estrecho. Dentro del marco del acuerdo, Malasia coordinó una series de ejercicios de guerra en red (network-centric warfare) en su zona económica exclusiva integrando elementos navales, aéreos y militares de sus países vecinos a través de una sofisticada plataforma de comunicaciones, control, y mando. Como resultado de estas operaciones, en agosto de 2006 el Comité de Guerra de Lloyd's anunció que excluía al Estrecho de Malaca de su Lista notando que 'el Comité opina que no solo es evidente que la situación ha mejorado en la región sino que también los esfuerzos adelantados son de largo plazo' (Joint War Committee, 2006).

El caso de Malaca y su inclusión en la Lista de Guerra es un ejemplo del poder que actores no tradicionales como Lloyd's ejercen a nivel global. En este caso Lloyd's asumió una política general frente al fenómeno de la piratería en una zona de importancia geoestratégica para el comercio global. En lugar de incluir restricciones específicas en las pólizas suscritas en su mercado, aunque ésta fue la consecuencia de la inclusión de la región en la Lista, la acción conjunta del Comité influenció la cooperación naval y militar de los está dos del litoral. Dicha cooperación había sido sugerida en anos anteriores por los Está dos Unidos, Japón y China, sin resultados tangibles. Interpretando el problema como una amenaza contra sus intereses nacionales, los Está dos Unidos y Japón llegaron incluso a ofrecerse a patrullar la zona lo cual fue interpretado, dentro de la misma lógica por los países del litoral, como una intervención en su soberanía. Asumiendo 
una racionalidad de riesgo, y por medio de un instrumento técnico, Lloyd's ejerció una forma de poder que incrementó los costes de circulación a través de la región con consecuencias económicas y políticas de gran importancia para los países del litoral. Está decisión fue el resultado de problematizar el fenómeno de la piratería en la región como un asunto del incremento en el riesgo de circulación, un problema técnico-actuarial que buscaba contrarrestarse el incremento del riesgo de acciones piraticas por medio de un incremento en el coste de las pólizas. En ultimas, al problematizar la piratería como una obstrucción a la circulación global, Lloyd's logró influenciar una decisión de está dos soberanos que de acuerdo a una lógica militar no habría sido posible. Aunque las dos racionalidades buscaban modificar la situación de seguridad en la región, el problematizar el fenómeno en términos de riesgo y no de amenaza logró una solución favorable, tanto en términos lucrativos como políticos.

De forma más reciente, y como ejemplo de su mayorazgo de la circulación marítima global, Lloyd's ha intentado nuevamente ejercer presión para establecer un orden de circulación marítima sobre el Océano Índico y particularmente en las costas del Golfo de Adén. El 16 de diciembre de 2010, el Comité de Guerra añadió a su Lista la región del Océano Índico comprendida entre un cuadrángulo enmarcado por el norte de Madagascar y el sur de la India cubriendo casi la totalidad de las rutas navegables hacia el Golfo de Adén y la Ciudad del Cabo en Suráfrica (Joint War Committee, 2010). Somalia venía siendo incluida en la Lista desde el 2005 y a lo largo de los cinco anos en los que ha operado el sistema de decisiones que opera el Comité de Guerra (ver Lobo-Guerrero, 2008) países vecinos y porciones del Océano Índico han venido agregándose. En contraste con la situación geopolítica del Estrecho de Malaca, no es mucho lo que Lloyd's puede hacer en este caso con relación a la conducta de Está dos soberanos. La Está dos de la costa oriental africana, particularmente en el caso de Somalia, carecen de los recursos, las capacidades y la organización para controlar la seguridad de las costas y de los mares territoriales. El fenómeno de la piratería en el Océano Índico ha sido problematizado por Lloyd's, en este caso, de forma distinta. El énfasis está siendo puesto en influir sobre la conducta de los propietarios de los buques y en la de los capitanes de los barcos para que acaten medidas acordadas por organizaciones interesadas en mantener un flujo marítimo ininterrumpido en las rutas mercantes.

Desde el ano 2008, la industria marítima internacional viene desarrollando documentos sobre 'mejores prácticas de gestión' (Best Management Practices -BMP) para la protección contra la piratería marítima originada en Somalia. En los documentos se dictan los procedimientos y tácticas a seguir para evitar ataques piratas, repelerlos una vez han empezado, y el accionar de los patrones y la tripulación si los piratas abordan el buque. En el 2009 se publicó la segunda versión (BMP2), en 2010 BMP3, y en 2011 BMP4. Los documentos están basados en tres requerimientos esenciales. El primero es que los operadores de los buques registren el movimiento de las naves con el Centro de Seguridad Marítima del Cuerno del África (MSCHOA por sus siglas en ingles) antes de ingresar en la zona de riesgo. ${ }^{4} \mathrm{El}$ MSCHOA es un centro de coordinación para facilitar la navegación segura y fue establecido de acuerdo al mandato de las Naciones Unidas. Lo opera un grupo multinacional con personal militar y de la industria mercante y tiene como función coordinar con las fuerzas navales y militares que operan en la zona la protección y el apoyo a las naves en tránsito (BMP3, 2011: 83). El segundo requerimiento es que una vez la nave entre a la zona de riesgo, debe reportarse su posición a la oficina regional en Dubái de la oficina de Operaciones de Comercio Marítimo del Reino Unido (UKMTO). Está entidad opera como el principal punto de contacto entre buques mercantes y las fuerzas militares en la región y administra un esquema voluntario por medio del cual los operadores marítimos registran la posición/curso/velocidad y hora estimada de llegada del buque al próximo puerto mientras se transita por la región de riesgo. Con está información la UKMTO rastrea el avance de la nave y coordina con el cuartel general de la Unión Europea cualquier rescate. También transmite información importante directamente a las naves lo cual evita tener que hacerlo con los operadores y propietarios y ahorra tiempo en caso

${ }^{4}$ La zona de riesgo está definida entre Suez y el Estrecho de Ormuz hacia el norte, $10^{\circ} \mathrm{S}$ y $78^{\circ} \mathrm{E}$. 
de una emergencia (BMP3, 2011: 85). El tercer requerimiento es que los operadores implementen las medidas de protección descritas en detalle en el documento BMP4, como se mencionará a continuación. El documento advierte que 'experiencia y datos recogidos por las fuerzas navales/militares indican que la aplicación de las recomendaciones contenidas en el folleto crean una significativa diferencia en la prevención de un ataque pirata' . Luego añade, '[1] as potenciales consecuencias de no seguir las recomendaciones de BMP son severas. Ha habido casos en los que los piratas han maltratado y violentado a sus rehenes' (BMP3, 2011: 1).

Las medidas de BMP son bastante precisas y vale la pena mencionar algunas de ellas ya que detallan el nivel al que se opera sobre la conducta de operadores, capitanes y tripulaciones. Dentro de las medidas de BMP se espera que los operadores realicen un análisis de riesgo para determinar la probabilidad y las consecuencias de un ataque pirata antes de ingresar en la zona de riesgo. Este análisis debe estar basado en la más reciente información proporcionada por las organizaciones mencionadas anteriormente, además de otras sugeridas en el documento. El resultado de este análisis debe ser la identificación de medidas de prevención, mitigación y recuperación incluyendo medidas estipuladas en está tutos concernientes al caso al igual que medidas suplementarias para combatir el fenómeno (BMP3, 2011: 5). Se recomienda que el análisis se refiera de forma particular a la nave y el recorrido planeado y no a generalidades ya que la situación en la zona de riesgo está en permanente evolución. Aspectos a ser considerados deben incluir la seguridad de la tripulación, particularmente la protección de las salidas de emergencia en caso de un incendio durante un ataque para evitar que el personal quede atrapado. También se recomienda prestar atención a la ubicación de la 'citadela', un recinto cerrado al interior de la nave con protección balística en el cual se puede refugiar la tripulación en caso de un abordaje pirata y desde el cual se pueden operar la maquinaria y las comunicaciones del buque de forma alterna. Se recomienda además proporcionar protección balística al personal que deba permanecer en el puente del buque durante un ataque ya que se sabe que los piratas dirigen sus disparos allí con el objetivo de intentar detener el barco (BMP3, 2011: 5). En términos de protección física, se sugiere que la altura mínima del resguardo de la nave sea de ocho metros para hacer más difícil un abordaje. En cuanto a la velocidad, se sugiere que en caso de detectarse un ataque se acelere a 18 nudos náuticos ya que hasta el momento ningún ataque ha sido efectivo a está velocidad aunque las lanchas utilizada por los piratas son capaces de alcanzar hasta 25 nudos. Se recomienda también maniobrar ya que dificulta un abordaje. En cuanto a las medidas para la protección de la nave las prácticas recomendadas incluyen, por ejemplo, que el personal de guardia disponga de la información y entrenamiento requerido, que cuente con material apropiado como lo son binoculares con capacidad de visión nocturna, que las guardias se roten con frecuencia y cuando sea posible que se refuercen con personal adicional, y que las lecturas del radar sean frecuentes. Se llega incluso a sugerir el instalar maniquíes de observadores para disuadir a los piratas. Se sugiere reforzar la seguridad del puente, ya que será el primer blanco de ataque, con elementos que minimicen los efectos de disparos de rockets utilizados por los piratas. Se recomienda el uso de concertina para proteger áreas vulnerables, mallas o barreras electrificadas con alto voltaje, y formas de canon de agua para repeler a los atacantes y demorar el abordaje. Dentro de estos se incluyen el uso de espumas y agua hirviendo. El manual enfatiza que la tripulación debe haberse entrenado en el uso de todas las medidas mencionadas y ejercicios periódicos deben asegurar que cada persona abordo sepa qué hacer en caso de un ataque. Las medidas se complementan con el uso de alarmas, circuitos cerrados de televisión para monitorear las distintas zonas del buque, y el mantener herramientas y elementos que puedan ser utilizadas por los atacantes debidamente guardadas o protegidas (p.e. tanques de gas o material inflamable).

El manual hace hincapié en que las medidas de BMP pueden contribuir a disminuir la posibilidad de un ataque, y en caso de que este suceda, demorar el abordaje lo cual daría tiempo a un rescate militar tenga lugar. Tal rescate requeriría que toda la tripulación se encuentre concentrada en la citadela de seguridad y que desde allí se puedan recibir y transmitir mensajes. Sin embargo, el documento reitera que un rescate no se puede garantizar, incluso cuando se hayan seguido todas las medidas de precaución.

La implementación de las medidas incluidas en 
los documentos BMP se está convirtiendo, aunque no de forma general, en condición de aseguramiento dentro de los contratos de seguro de guerra que cubren la piratería sobre los buques que transitan a través de la zona de riesgo. Al estar la zona de riesgo incluida en la Lista del Comité de Guerra de Lloyd's, los contratos de seguro suscritos en ese mercado que cubren cerca del 70 por ciento del transito en la zona- incluyen una clausula que limita la navegación por esas zonas. Esto obliga a los propietarios de los buques a negociar con los aseguradores una póliza especifica que les provea protección durante el tiempo que dure el recorrido por la zona excluida. Los aseguradores sugieren al asegurado adoptar las medidas de BMP4. De esta forma, las medidas sugeridas por las organizaciones del comercio marítimo internacional en los documentos descritos anteriormente se convierten en prescripciones del comportamiento de operadores y tripulaciones de las naves que transitan por la zona. Esto es, por supuesto, si han de navegar con seguro, lo cual no es obligatorio pero la ausencia de seguro representa un riesgo demasiado alto a ser cubierto de forma individual.

\section{CONCLUSIONES}

El analizar los seguros marítimos y el rol de Lloyd's of London como tecnología y agente de poder internacional que contribuyen a la producción de la seguridad oceánica permite replantear la espacialidad de los mares y de los océanos por fuera de los lineamientos territoriales tradicionales y posicionarla en el área de la biopolitica de la movilidad. Esto, a la vez, permite analizar la movilidad marítima desde el cuasi-transcendente de la circulación, una categoría que facilita la revelación del fenómeno de la movilidad y la seguridad como efectos de complejos entramados de poder. Estos incluyen no solo las relaciones de producción y consumo de las economías globales, sino también las estrategias de administración de riesgo desarrolladas para controlar la incertidumbre alrededor de la navegación marítima.

A partir de la proyección de los imaginarios de riesgo sobre los cuales operan los suscriptores dentro del mercado de Lloyd's, y teniendo en cuenta los efectos de las condiciones impuestas por los aseguradores sobre los asegurados en términos de la con- ducta de los últimos, es posible argumentar que lo que se proyecta en último término es una economía moral que a su vez expresa una forma particular de vida. Esta forma de vida está determinada por la capacidad de transformar incertidumbres en material fungible, entendido como riesgo, y de utilizar tal riesgo como elemento operador de lo que puede llamarse, siguiendo una tradición Foucaulteana, una biopolítica de seguridad. Tal biopolítica de seguridad asume como problema, no las amenazas a un sistema, sino la incertidumbre que subyace la capacidad de circular ininterrumpidamente por los océanos. Esta forma de vida, que bien puede llamarse, haciendo una gran generalización, como liberal occidental, depende de la capacidad de generar formas de compensación por la pérdida de capital, bien en la forma de pérdida de buques y carga, pero también en la pérdida de la capacidad de circular como recurso vital para el comercio global.

Dos elementos generales para la teoría biopolitica se derivan de este análisis. El primero es la construcción de la piratería como forma excluyente y amenazante frente a una comunidad política cerrada y acorazada ante el riesgo y la incertidumbre. La piratería representa en principio una amenaza a las formas de circulación oceánica de las que depende la vida liberal occidental. Sin embargo, la comunidad política liberal no problematiza la piratería empleando una lógica de suma-cero como se ha hecho de forma tradicional cuando se utiliza la fuerza militar. En este caso el asunto se ha problematizado en términos de riesgo utilizando una lógica que permite transformar la incertidumbre en materia de gobernanza y gestión. Por medio de los seguros marítimos, y no a través de prácticas tradicionales del poder soberano como lo son la defensa y la diplomacia, se ha interpretado y representado a la piratería como una obstrucción a la movilidad, como un problema operacional en la circulación oceánica. Como tal, la piratería no adquiere dimensión humana, ni social, ni política. Se presenta de manera simplificada como una forma de vida que en tanto obstaculiza la movilidad marítima debe ser neutralizada. Su neutralización es operada a través de instrumentos de riesgo como lo son los seguros marítimos. El riesgo opera en este caso como la racionalidad a través de la cual se busca 'gestionar' los efectos adversos de una forma de vida que existe 
de forma paralela a la liberal occidental. Tal neutralización no implica ni requiere de su destrucción. La promoción y protección de la forma de vida liberal occidental se hace posible al reducir la problemática pirática a un asunto de administración de riesgos, no un problema tradicionalmente político sino uno que se presenta en términos industriales/operacionales.

El segundo elemento se desprende del anterior y se refiere a un fenómeno bien conocido dentro de la teoría biopolitica. Se trata de la necesidad que tienen los regímenes biopoliticos de producir, crear, e identificar a un enemigo como condición de su posibilidad. Si bien es cierto que los regímenes biopoliticos se han analizado generalmente desde la agencia de los gobiernos y de las formas de poder soberano que estos ejercen, como se ve en el trabajo de Agamben y hasta cierto punto de Esposito, el material y el caso presentado en este artículo hacen evidente la operación de regímenes biopoliticos que transcienden la esfera meramente gubernamental. En el régimen estudiado en este artículo, la categoría de enemigo es entendida de manera distinta. El 'enemigo' bajo una racionalidad de riesgo no es el enemigo del que trata Schmitt en su definición de lo político (Schmitt, 2007). No es entendido como un elemento que presenta una amenaza existencial y que por lo tanto debe destruirse, o dejarse morir. Es un elemento que se asume como reto de gestión, que como función de una lógica de riesgo puede administrarse por medio de tecnologías que transforman la incertidumbre en objeto de transacción. El enemigo en este caso cumple la función de elemento facilitador en la constitución de un mercado de riesgos, mercados que operan a través de productos como los seguros marítimos.

La utilización de los seguros marítimos como instrumentos para facilitar la seguridad de la movilidad oceánica no es por tanto una práctica inocente. Involucra un complejo entramado de poder que procede a partir de la implementación de una racionalidad de riesgo. Tal racionalidad requiere para su operación de un imaginario que permite entender la incertidumbre como un objeto fungible y que al ser utilizada en la gobernanza oceánica constituye una biopolitica de movilidad que bien merece ser estudiada en detalle.

\section{BIBLIOGRAFÍA}

Agamben, G. 1998. Homo Sacer, Sovereign Power and Bare Life, Stanford, Stanford University Press.

BAKER, T. 2000. Insuring Morality. Economy and Society, 29, 559-577.

BAKER, T. \& SimON, J. 2002. Embracing Risk. In: BAKER, T. \& SIMON, J. (eds.) Embracing Risk: The Changing Culture of Insurance and Responsibility. Chicago: University of Chicago Press 1-26.

Barry, A. 2001. Political Machines: Governing a Technological Society, London and New York, Athlone Press.

BBC News 2011. Somali piracy: Armed guards to protect UK ships. http://www.bbc.co.uk/news/uk15510467, consultado: 31 Octubre 2011.

BMP3 2011. Best Management Practices for Protection against Somalia Based Piracy, Edinburgo, Witherby Publishing.

BurCheLL, G. 1996. Liberal government and techniques of the self. In: ANDREW BARRY, T. O., NIKOLAS ROSE (ed.) Foucault and Political Reason: Liberalism, neo-liberalism and rationalities of government. London: University College London 19-36.

Clark, G., Anderson, G., Thomann,C. \&Graf Von der Schulengurg, J.M. 2010. The Appeal of Insurance, Toronto, University of Toronto Press.

Dean, M. 1999a. Governmentality: Power and Rule in Modern Society, London, Sage.

DeAn, M. 1999b. Risk, Calculable and Incalculable In: LUPTON, D. (ed.) Risk and Socio-Cultural Theory. Cambridge: Cambridge University Press.

Dillon, M. \& Lobo-Guerrero, L. 2009. The Biopolitical Imaginary of Species Being. Theory Culture Society, 26, 1-23.

Ericson, R., Doyle, A. \& BARry, D. 2004. Insurance as Governance, Toronto, University of Toronto Press. EsCRICHE, J. 1840. Elementos del derecho español. Paris: Libreria de Vicente Salva 106-114. 
Esposito, R. 2012. El Dispositivo de la Persona, Madrid, Buenos Aires, Amorrortu Editores.

Ewald, F. 1991. Insurance and Risk. In: Burchell, G., Gordon, C. \& Miller, P. (eds.) The Foucault Effect: Studies in Governmentality, with two lectures and an interview with Michel Foucault. Chicago: The University of Chicago Press 197-210.

Foucault, M. 1964. Madness and Civilization, Pantheon Books, New York.

Foucault, M. 1989. The Order of Things, London, Routledge.

Foucault, M. 1991. Discipline and Punish: the Birth of the Prison, London, Penguin Books.

Foucault, M. 1997. De los Espacios Otros. http://textosenlinea.blogspot.com/2008/05/michel-foucault-losespacios-otros.html, «Des espaces autres», conferencia pronunciada en el Centre d'Études architecturales el 14 de marzo de 1967 y publicada en Architecture, Mouvement, Continuité, n 5, octubre 1984, págs. 46-49. Traducción al español por Luis Gayo Pérez Bueno, publicada en revista Astrágalo, $\mathrm{n}^{\circ} 7$, septiembre de 1997.

Foucault, M. 1998 The History of Sexuality Vol. 1: the Will to Knowledge, London, Penguin.

Foucault, M. 2001. Dits et Ecrits, 4 vols., Paris, Galimard.

Foucault, M. 2003. Society Must Be Defended, London, Penguin Books.

Foucault, M. 2004a. The Archeology of Knowledge London, Routledge.

Foucault, M. 2004b. Sécurité, Territoire, Population, 1977-1978, Lectures au Collège de France, Paris, Hautes Etudes, Seuil Gallimard.

Foucault, M. 2008. The Birth of Biopolitics, London, Palgrave Macmillan.

GiBB, D. E. W. 1957. Lloyd's of London: A Study in Individualism, London, Macmillan and Co. Ltd. .

Hardt, M. \& Negri, A. 2001. Empire, Cambridge, Mass. ; London, Harvard University Press.

Hardt, M. \& Negri, A. 2004. Multitude : war and democracy in the age of Empire New York, The Penguin Press.

Harvard Law Review 1920. Marine Risk and War Risk. Harvard Law Review, 33, 706-709.

Haufler, V. 1993. Crossing the Boundary between Public and Private: International Regimes and Non-State Actors. In: RITTBERGER, V. (ed.) Regime Theory and International Relations. Oxford: Clarendon Press 94-111.

Haufler, V. 1997. Dangerous Commerce: Insurance and the management of international risk, Ithaca and London, Cornell University Press.

House of Lords EU Comittee 2010. 12th Report of Session 2009-2010 - Combating Somali Piracy: the EU's Naval Operation Atalanta, Report with Evidence, London, The Stationery Office.

IMB 2011. International Maritime Bureau Piracy Reporting Centre. http://www.icc-ccs.org/piracy-reporting-centre.

Joint War Committe 2006. JWC Hull War, Strikes, Terrorism and Related Perils Listed Areas. Lloyd's Market Association, JWLA005, 7 August.

Joint War Committe 2010. JWC Hull War, Strikes, Terrorism and Related Perils Listed Areas. Lloyd's Market Association, JWLA016, 16 December 2010.

Leander, A. 2010. The Paradoxical Impunity of Private Military Companies: Authority and the Limits to Legal Accountability. Security Dialogue, 41, 467-490.

LLOYD's OF LONDON 2011. Piracy: high crime in the high seas. News and Features, http://www.lloyds.com/ News-and-Insight/News-and-Features/Geopolitical/Geopolitical-2011/Piracy-High-crime-on-the-highseas, visitado el 31 de Octubre de 2011.

Lobo-Guerrero, L. 2008. "Pirates," Stewards, and the Securitization of Global Circulation. International Political Sociology, 2, 219-235.

Lobo-Guerrero, L. 2011. Insuring Securtity: Biopolitics, Security and Risk, Abingdon, Ox., Routledge.

Lobo-Guerrero, L. 2012a. Insuring War: sovereignty, security and risk, Abingdon, Ox., Routledge.

LoBo-Guerrero, L. 2012b. Lloyd's and the moral economy of insuring against piracy: towards a politicisation of marine war risks insurance. Journal of Cultural Economy, 5. 
Marchal, R. 2011. Somali Piracy: The Local Contexts of an International Obsession. Humanity Journal, 2, 31-50.

Mendiola Gonzalo, I. (ed.) 2009. Rastros y rostros de la biopolitica, Barcelona: Anthropos.

Miu, L. S. 2007. Seaborne Trade Accounts for 77\% of Total World Trade. Lloyd's MIU.

Murphy, M. N. 2010. Small Boats, Weak States, Dirty Money: Piracy and Maritime Terrorism in the Modern World, New York, Columbia University Press.

O’Malley, P. 2004. Risk, Uncertainty and Government, London, Glasshouse Press.

Odeke, A. 2011. Somali Piracy - Effects on Oceanborne Commerce and regional security and challenges to international law and World order. Australian and New Zealand Maritime Law Journal, 25, 134-160.

PARK, J. A. 1817. A System of the Law of Marine Insurances With Three Chapters on Bottomry, on Insurances on Lives, on Insurances on Fire London, J. Butterworth and Son.

Power, M. 2003. Risk Management and the Responsible Organisation. In: Ericson, R. \& Doyle, A. (eds.) Risk and Morality. Toronto: University of Toronto Press 145-164.

Power, M. 2004. The Risk Management of Everything: Rethinking the politics of uncertainty, London, Demos.

Power, M. 2007. Organised Uncertainty: Designing a World of Risk Management, Oxford, Oxford University Press.

Rose, F. D. 2004. Marine Insurance: Law and Practice, London, Informa Professional.

Rose, N. 1999. Powers of Freedom, Cambridge, Cambridge University Press.

Schmitt, C. 2003. The Nomos of the Earth in the International Law of the Jus Publicum Europeaum, New York, Telos Press Publishing.

Sснмітт, C. 2007. The Concept of the Political: Expanded Edition, Chicago, University of Chicago Press.

Schmitt, C. 2011. The Concept of Piracy. Humanity Journal, 2, 26-30.

Steinberg, P. 2001. The Social Construction of the Ocean, Cambridge, Cambridge University Press.

Trenerry, C. F. 1926. The Origin and Early History of Insurance, Including the Contract of Bottomry, London, P.S. King \& Son, Ltd.

WeSketT, J. 1781. A complete digest of the theory, laws, and practice of insurance, London, Richardson \& Urquhart.

Wright, R. 2011. Daunting challenge of pirates' growing range. The Financial Times, Febrero 14, 2011, http://www.ft.com/cms/s/0/34c7af5c-386a-11e0-959c-00144feabdc0.html - axzz1cMpVeFDt, consultado: 31 Octubre 2011.

Young, A. 2007. Contemporary Piracy in Southeast Asia: History, Causes, and Remedies, Amsterdam, International Institute for Asian Studies, The Netherlands - Institute of Southeast Asian Studies, Singapore. 